Terreno, D., Pérez, J. y Sattler, S. (2020). La relación entre liquidez, rentabilidad y solvencia: Una investigación empírica por el modelo de ecuaciones estructurales. Contaduría Universidad de Antioquia, 77, 13-35.

Doi: https://doi.org/10.17533/udea.rc.n77a01

\title{
La relación entre liquidez, rentabilidad y solvencia: Una investigación empírica por el modelo de ecuaciones estructurales
}

Dante Domingo Terreno

Universidad Católica de Córdoba

danteterreno@fibertel.com.ar orcid: 0000-0003-4400-8058

Jorge Orlando Peréz Universidad Católica de Córdoba jorgeoperez53@gmail.com orcid: 0000-0001-5974-6024

Silvana Andrea Sattler Universidad Católica de Córdoba silsattler@gmail.com orcid: 0000-0002-9900-9085 
La relación entre liquidez, rentabilidad y solvencia: una investigación empírica por el modelo de ecuaciones estructurales

Resumen: El objetivo del estudio fue determinar la relación de la liquidez con la rentabilidad y solvencia en empresas que cotizan en el Mercado de Valores de Buenos Aires, por el Modelo de Ecuaciones Estructurales. Los resultados indican que las empresas con mayor capital corriente tienen una mayor rentabilidad, lo cual obedece a una actitud cortoplacista del empresario argentino donde la mayor inversión es efectuada en inventarios. La relación entre liquidez y el endeudamiento a largo plazo es negativa, las empresas con mayor endeudamiento de largo plazo tienen un menor capital corriente. Lo cual obedece a que el costo de los pasivos obliga a reducir al mínimo el activo corriente y, además, las empresas con necesidad de financiamiento utilizan fuentes de largo y corto plazo.

Palabras clave: Liquidez, rentabilidad, endeudamiento, SEM; inventarios.

The relationship between liquidity, profitability and solvency: empirical research through the structural equations model

Abstract: This study aimed at determining the relationship between liquidity and the profitability and solvency of the firms listed on the Buenos Aires Stock Exchange, through the Structural Equations Model. The results indicate that firms with greater current capital have greater profitability, which results from a short-termist attitude of the Argentinian business owner due to which the greatest investment is made in inventories. The relationship between liquidity and long-term indebtedness is negative; firms with greater long-term indebtedness have lower current capital. This is because the cost of liabilities forces a reduction of current assets to the minimum; besides, firms with financing needs use long-term and short-term sources.

Keywords: Liquidity, profitability, indebtedness, SEM; inventories.

A relação entre liquidez, rentabilidade e solvência: Uma pesquisa empírica por Modelagem de Equações Estruturais

Resumo: O objetivo do estudo foi determinar a relação da liquidez com a rentabilidade e a solvência em empresas que têm cotação no Mercado de Valores de Buenos Aires, por Modelagem de Equações Estruturais. Os resultados evidenciam que as empresas com maior capital de giro têm uma maior rentabilidade, o que obedece a uma atitude curtoprazista do empresário argentino onde o maior investimento é feito em estoque. A relação entre liquidez e o endividamento a longo prazo é negativa, as empresas com maior endividamento de longo prazo têm menor capital de giro. Devido a que o custo dos passivos obriga a reduzir ao mínimo o ativo corrente e, além disso, as empresas com necessidade de financiamento utilizam fontes de longo e curto prazo.

Palavras chave: Liquidez, rentabilidade, endividamento; SEM, estoque.

Le rapport entre liquidité, rentabilité et solvabilité : une recherche empirique par le modèle d'équations structurales

Résumé: Le but de cette étude a été de déterminer le rapport entre la liquidité et la rentabilité et la solvabilité dans les entreprises qui cotent dans le Marché de Valeurs de Buenos Aires en Argentine, par le Modèle d'Équations Structurales. Les résultats démontrent que les entreprises avec plus de capital courant ont une plus haute rentabilité, ce qui obéit à une attitude courtermiste de l'entrepreneur argentin où le plus grand investissement est fait sur les inventaires. Le rapport entre liquidité et endettement à long terme est négatif, les entreprises avec plus d'endettement de long terme ont un capital courant réduit; du fait que le coût des passifs oblige à reduire au minimum l'actif courant et, en plus, les entreprises necessitant de financement font appel à des sources de long et court terme.

Mots clés: liquidité, rentabilité, endettement, SEM, inventaires. 
Cont. udea (julio-diciembre), pp. 13-35. (C) Universidad de Antioquia-2020.

\title{
La relación entre liquidez, rentabilidad y solvencia: una investigación empírica por el modelo de ecuaciones estructurales
}

\author{
Dante Domingo Terreno, Jorge Orlando Pérez y Silvana Andrea Sattler \\ https://doi.org/10.17533/udea.rc.n77a01
}

Primera versión recibida en enero de 2020 - Versión aceptada en junio de 2020

\section{Introducción}

Cl objetivo de la administración del capital corriente es lograr un óptimo Eentre liquidez y rentabilidad de la empresa. El óptimo del capital corriente (o capital de trabajo) se alcanza cuando se logra maximizar la necesidad de liquidez y la rentabilidad (Brealey, Myers y Allen, 2006). El capital de trabajo o fondo de maniobra debe cubrir las necesidades operativas sin caer en el problema de liquidez, ni tampoco en un exceso que distraiga recursos de otras inversiones. Las decisiones relativas al capital de trabajo conllevan dos tipos de decisiones, las vinculadas a las necesidades operativas y a la financiación. En el análisis de estados financieros, habitualmente el estudio de la administración del capital de trabajo es conocido como liquidez, y como la empresa es un todo, es necesario establecer la vinculación con los sectores de la rentabilidad y solvencia.

Una modelización simple de la relación entre los componentes de los estados financieros, a priori, indican una relación negativa de la liquidez con la rentabilidad y una relación positiva entre liquidez y endeudamiento de largo plazo, pero la evidencia empírica no lo corrobora en todos los casos. Algunos estudios confirman la existencia de un trade-off (relación inversa) entre liquidez y rentabilidad (Eljelly, 2004; Raheman y Nasr, 2007; Raykov, 2017), empresas con mayor capital corriente tienen un menor nivel de rentabilidad. Pero existen otras evidencias que indican una relación positiva entre liquidez y rentabilidad (Afza y Nazir, 2007; Vazquez Carrazana, Rech, Miranda y Tavares, 2017; Kadayifci y Coskun, 2017). Por otra parte, otros estudios (Azeem y Marsap, 2015; Kadayifci y Coskun, 2017) encuentran que las 
Terreno, D., Pérez, J. y Sattler, S. La relación entre liquidez, rentabilidad y solvencia...

empresas con mayor endeudamiento tienen un menor capital de trabajo; sin embargo, hay evidencias que muestran una relación positiva (Gill, 2011; Naser, Nuseibeh y Al-Hadeya, 2013).

En la bibliografía, los ratios más utilizados para la medición del capital corriente son el índice de liquidez corriente (Lazaridis y Tryfonidis, 2006), el ciclo de conversión de efectivo (Palombini y Nakamura, 2012; Deloof, 2003), el capital corriente requerido (Gill, 2011; Afza y Nazir, 2007) y la liquidez neta de equilibrio (Chiou y Cheng, 2006). En realidad, estos ratios no se contraponen, cada uno analiza aspectos diferentes de liquidez. Por lo tanto, es importante el estudio por la técnica estadística del Modelo de Ecuaciones Estructurales (Structural Equation Modeling, SEM) que analizan la liquidez, rentabilidad y solvencia como un constructo formado por un conjunto de ratios contables. Esto permite un análisis integral y de las interrelaciones de los ratios, a diferencia de la simple correlación entre variables del método de mínimos cuadrados ordinarios.

Por otra parte, si bien la mayor parte de las investigaciones actuales han sido llevadas a cabo en economías emergentes, no se han efectuado estudios de este tipo para la Argentina, una economía turbulenta con crisis recurrentes, un importante nivel de inflación y escasez del crédito. Es por ello, que el objetivo del estudio es determinar la relación de la liquidez con la rentabilidad y solvencia en empresas que cotizan en el Mercado de Valores de Buenos Aires, por el Modelo de Ecuaciones Estructurales. La relación entre dichos sectores es también analizada por los cambios anuales de los ratios. De esta manera, el presente trabajo intenta ser un aporte para los usuarios de los estados financieros y administradores.

\section{Marco teórico}

Como punto de partida, es conveniente efectuar una definición de los principales conceptos utilizados en este trabajo. Por administración del capital de trabajo, se entiende a la estrategia que tiene por objetivo una eficiente administración de los activos y pasivos corrientes. Siendo la relación entre la rentabilidad y riesgo la clave de la administración del capital de trabajo. La liquidez es entendida como la flexibilidad para cambiar activos por otros más líquidos, en un contexto en el que esta asegure el pago de las obligaciones de la empresa.

Por otra parte, un concepto central en este trabajo es el de capital corriente, se lo puede definir como los recursos necesarios para llevar adelante las operaciones de la empresa, medido por la diferencia de activos y pasivos corrientes. El capital corriente puede ser dividido en capital corriente operacional (capital corriente requerido) y financiero (liquidez neta de equilibrio) (Shulman y Cox's, 1985): 
a) Capital Corriente Requerido (RCC): se define como la diferencia entre los activos corrientes operacionales, tales como créditos por ventas, bienes de cambio y pasivos corrientes operacionales (como las deudas comerciales). El capital corriente requerido es determinado por:

- el ciclo de conversión de efectivo (plazo de cobranza, plazo de almacenamiento y plazo de pagos) y;

- por el nivel de actividad (ventas, costo de ventas, compras).

b) Liquidez neta de equilibrio (LNE): es la diferencia entre el capital corriente actual y el capital corriente requerido. La cual incluye caja y bancos, e inversiones transitorias, menos deudas financieras de corto plazo y deudas financieras de largo plazo con vencimiento en el corto plazo (Shulman y Cox's, 1985). La figura $\mathrm{N}^{\circ} 1$ ilustra la relación entre RCC y LNE.

La liquidez corriente es una de las medidas más tradicionales del capital corriente de la empresa y relaciona los activos corrientes con los pasivos corrientes. El requerimiento de capital corriente y la liquidez neta de equilibrio dividido por el total de activos, o la relación entre activos y pasivos, pueden ser otras medidas de liquidez.

Figura 1: Capital corriente requerido y liquidez neta de equilibrio

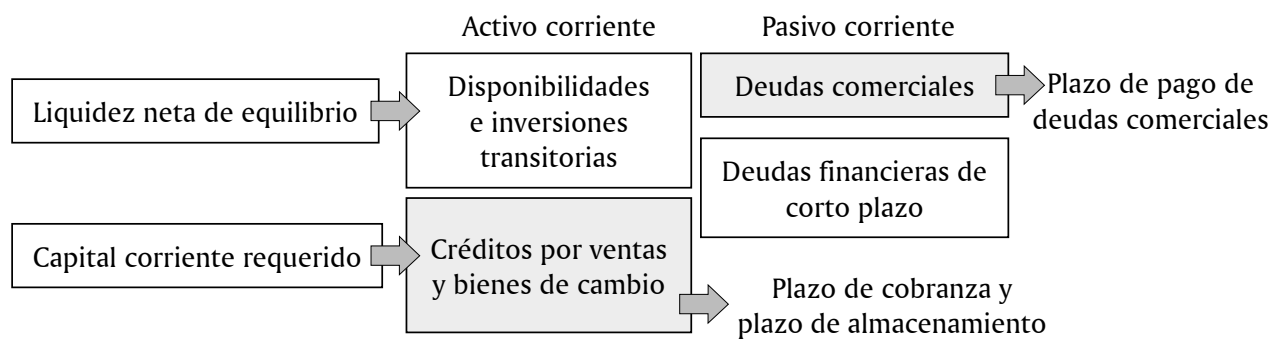

Fuente: Elaboración propia.

El ciclo de conversión de efectivo (CCE) representa el número de días en que los fondos de la empresa en promedio son invertidos en capital de trabajo (Gitman, 1982). El CCE es el resultado de ciclo operativo que realiza la empresa para llevar adelante las actividades (comprar, producir y vender).

Una gran parte de los estudios sobre administración de capital de trabajo se han enfocado en el ciclo de conversión de efectivo, como medida de la eficacia en la gestión del capital de trabajo, y han encontrado una relación negativa con el nivel de rentabilidad (Palombini y Nakamura, 2012; Gill, Biger y Mathur, 2010; García-Teruel y Martínez-Solano, 2007; Deloof, 2003; Shin y Soenen, 1998). Shin y Soenen (1998) analizan la relación entre el ciclo de conversión en efectivo y la rentabilidad de una muestra de empresas de Estados Unidos durante el período 1974-1994. Los resultados indican que si se reduce el ciclo de conversión de 
Terreno, D., Pérez, J. y Sattler, S. La relación entre liquidez, rentabilidad y solvencia...

efectivo se logra incrementar la rentabilidad de la empresa. Eljelly (2004) para Arabia Saudita encontró una relación negativa significativa entre la rentabilidad y su nivel de liquidez, medido por el índice de liquidez corriente. Sin embargo, a nivel de la industria, el estudio encontró que el ciclo de conversión de efectivo o brecha de efectivo es más importante como medida de liquidez. Deloof (2003) para empresas de Bélgica, durante el período 1992-1996, y García-Teruel y Martínez-Solano (2007) para pequeñas y medianas empresas de España confirman que las empresas pueden incrementar la rentabilidad reduciendo los días de cuentas a cobrar e inventarios. Naser, Nuseibeh y Al-Hadeya (2013) para empresas del mercado de valores de Abu Dhabi encuentran que las que tienen un alto ratio de endeudamiento tienden a tener un CCE largo, en comparación con empresas que tienen un bajo nivel de apalancamiento. Esto se explica porque las empresas que tienen un CCE largo deben recurrir a fuentes externas de financiamiento.

Los estudios sobre los determinantes del capital corriente (Azeem y Marsap, 2015; Chiou y Cheng, 2006) encuentran evidencias de una relación negativa entre el capital corriente requerido y la liquidez corriente con la rentabilidad, entre otras variables. Sin embargo, Kadayifci y Coskun (2017) y Afza y Nazir, (2007) muestran una relación positiva. Afza y Nazir, (2007) analizan para Pakistán la relación entre la rentabilidad y las medidas del grado de agresividad de las políticas de administración del capital de trabajo. La evidencia indica una relación positiva entre el capital corriente y el nivel de rentabilidad.

Los estudios sobre los determinantes del capital encuentran una relación negativa entre el nivel de capital corriente y endeudamiento (Kadayifci y Coskun, 2017 Azeem y Marsap, 2015; Wasiuzzaman y Arumugam, 2013). El aumento de la deuda, por los costos que ello implica, obliga a las empresas a administrar su capital de trabajo de manera más eficiente, por lo tanto, a reducir su inversión en capital de corriente. Chiou y Cheng (2006) investigan los determinantes del capital corriente a través del capital corriente requerido y la liquidez neta de equilibrio, por los incrementos anuales, y concluyen en la existencia de una relación negativa de la rentabilidad y endeudamiento. En otro estudio, Maradi, Salehi y Arianpoor (2012) de industria farmacéutica y química de Irán, encuentran una relación negativa entre el nivel de endeudamiento y la liquidez neta de equilibrio.

Mandujano Herrera y Navarro Orihuela (2015) analizan los determinantes del capital corriente del sector manufacturero de Perú y Chile. Los resultados indican una relación negativa del ciclo de conversión en efectivo y el requerimiento de capital de trabajo con la rentabilidad. En relación con el endeudamiento, en el caso chileno, el mayor nivel de deuda genera ciclos más largos; sin embargo, en Perú que posee un sistema bancario menos desarrollado 
y de más difícil acceso que el chileno, da lugar a una relación inversa entre ambas variables.

Baser, Gokten, Kucukkocaoglu y Ture (2016) examinan para Turquía la validez de la existencia de un trade-off entre rentabilidad y liquidez a través del modelado de ecuaciones estructurales, y verificaron la existencia de un moderado efecto positivo entre liquidez y rentabilidad. Saarani y Shahadan (2012) para Malasia, aplicando PLS-SEM (Mínimos Cuadrados Parciales - Modelo de Ecuaciones Estructurales), encuentran como factores determinantes del capital corriente a la rentabilidad y el endeudamiento. El primero tiene una relación positiva y el segundo negativa. Vazquez Carrazana y otros (2017) estudian 20 empresas del sector de agronegocio brasileño y verifican una correlación positiva y significativa entre la rentabilidad y la liquidez.

En síntesis, las evidencias no son concluyentes sobre la dirección de relación de la liquidez con la rentabilidad y el endeudamiento. Una de las posibles razones que explican las diferencias entre las investigaciones, es que el requerimiento de capital de trabajo y las prácticas de los administradores difieren entre industrias y entre países (Gill, 2011). En la tabla $\mathrm{N}^{\circ} 1$ se efectúa una síntesis de la revisión bibliografía.

Tabla 1: Revisión bibliográfica

\begin{tabular}{|c|c|c|c|c|c|c|c|c|}
\hline \multirow{2}{*}{ Autores } & \multirow{2}{*}{ Países } & \multicolumn{5}{|c|}{$\begin{array}{c}\text { Variable } \\
\text { dependiente }\end{array}$} & \multicolumn{2}{|c|}{ Variables independientes } \\
\hline & & Período & CCE & CCR & LC & LNE & Rentabilidad & Endeudamiento \\
\hline Shin y Soenen (1998) & USA & 1975-1994 & $\mathrm{X}$ & & & & Negativa & \\
\hline Deloof (2003) & Bélgica & $1992-1996$ & $\mathrm{X}$ & & & & Negativa & \\
\hline Eljelly (2004) & Arabia Saudita & $1996-2000$ & $\mathrm{X}$ & & $\mathrm{X}$ & & Negativa & \\
\hline $\begin{array}{l}\text { Lazaridis y Tryfonidis } \\
(2006)\end{array}$ & Grecia & $2001-2004$ & $\mathrm{X}$ & & & & Negativa & \\
\hline Chiou y Cheng (2006) & Taiwán & $1996-2004$ & & $\mathrm{X}$ & & $\mathrm{X}$ & Negativa & Negativa \\
\hline Afza y Nazir (2007) & Pakistán & $1998-2005$ & & & $\mathrm{X}$ & & Positiva & \\
\hline $\begin{array}{l}\text { García-Teruel y } \\
\text { Martinez-Solano (2007) }\end{array}$ & España & $1996-2002$ & $\mathrm{X}$ & & & & Negativa & \\
\hline Raheman y Nasr (2007) & Pakistán & $1999-2004$ & $\mathrm{X}$ & & $\mathrm{X}$ & & Negativa & \\
\hline $\begin{array}{l}\text { Zariyawati, Annuar, } \\
\text { Taufiq y Rahim (2009) }\end{array}$ & Malasia & $1996-2006$ & $\mathrm{X}$ & & & & Negativa & \\
\hline $\begin{array}{l}\text { Gill, Biger y Mathur } \\
\text { (2010) }\end{array}$ & Estados Unidos & $2003-2007$ & $\mathrm{X}$ & & & & Negativa & \\
\hline Gill (2011) & Canadá & $2008-2010$ & & $\mathrm{X}$ & & & Positiva & Positiva (") \\
\hline $\begin{array}{l}\text { Palombini y Nakamura } \\
\text { (2012) }\end{array}$ & Brasil & $2001-2008$ & $\mathrm{X}$ & & & & Negativa & Negativa \\
\hline
\end{tabular}


Terreno, D., Pérez, J. y Sattler, S. La relación entre liquidez, rentabilidad y solvencia...

\begin{tabular}{|c|c|c|c|c|c|c|c|c|}
\hline \multirow{2}{*}{ Autores } & \multirow{2}{*}{ Países } & \multicolumn{5}{|c|}{$\begin{array}{c}\text { Variable } \\
\text { dependiente }\end{array}$} & \multicolumn{2}{|c|}{ Variables independientes } \\
\hline & & Período & CCE & CCR & LC & LNE & Rentabilidad & Endeudamiento \\
\hline $\begin{array}{l}\text { Shubita y Alsawalhah } \\
\text { (2012) }\end{array}$ & Jordania & $2004-2011$ & & & $\mathrm{X}$ & & Negativa & \\
\hline $\begin{array}{l}\text { Maradi,Salehi y } \\
\text { Arianpoor (2012) }\end{array}$ & Irán (橉) & $2001-2008$ & & $\mathrm{X}$ & & $\mathrm{X}$ & & Negativa \\
\hline $\begin{array}{l}\text { Wasiuzzaman y } \\
\text { Arumugam (2013) }\end{array}$ & Malasia & $2000-2007$ & & $\mathrm{X}$ & & & & Negativa \\
\hline $\begin{array}{l}\text { Naser, Nuseibeh y Al- } \\
\text { Hadeya (2013). }\end{array}$ & Emiratos & $2010-2011$ & $\mathrm{X}$ & & & & Negativa & Positiva \\
\hline $\begin{array}{l}\text { Mandujano Herrera y } \\
\text { Navarro Orihuela (2015) }\end{array}$ & Chile y Perú & $2007-2013$ & $\mathrm{X}$ & & & & Negativa & (**) Posit/Neg. \\
\hline Azeem y Marsap (2015) & Pakistán & 2004-2009 & & & $\mathrm{X}$ & & Negativa & Negativa \\
\hline $\begin{array}{l}\text { Kadayifci y Coskun } \\
\text { (2017) }\end{array}$ & Turquía & $2011-2016$ & & & $\mathrm{X}$ & & Positiva & Negativa \\
\hline $\begin{array}{l}\text { Vazquez Carrazana y } \\
\text { otros (2017) }\end{array}$ & Brasil & $2005-2015$ & & & $\mathrm{X}$ & & Positiva & \\
\hline Raykov (2017) & Bulgaria & $2007-2015$ & & & $\mathrm{X}$ & & Negativa & \\
\hline
\end{tabular}

Fuente: Elaboración propia.

CCE: ciclo de conversión en efectivo; CCR: capital corriente requerido; LC: liquidez corriente; LNE: liquidez neta de equilibrio (") Positiva para Chile y Negativo para Perú; ("**) para la industria manufacturera.

\section{II.I. Economía argentina}

Tabla 2: Indicadores económicos

\begin{tabular}{ccc}
\hline Año & \%IPC & \%PBI \\
\hline 2005 & 12,30 & 8,90 \\
2006 & 9,80 & 8,00 \\
2007 & 8,50 & 9,00 \\
2008 & 7,20 & 4,10 \\
2009 & 7,70 & $-5,90$ \\
2010 & 10,90 & 10,10 \\
2011 & 9,50 & 6,00 \\
2012 & 10,80 & $-1,00$ \\
2013 & 26,60 & 2,40 \\
2014 & 38,00 & $-2,50$ \\
2015 & 26,90 & 2,70 \\
2016 & 42,20 & $-1,80$ \\
\hline
\end{tabular}

Fuente: Instituto Nacional de Estadística y Censos de la República Argentina, con excepción del IPC de los años 2013 a 2015 obtenidos por la Dirección General de Estadística y Censos de la

Ciudad de Buenos Aires. 
La Tabla 2 informa sobre la inflación y el crecimiento del Producto Bruto Interno (PBI). Los datos de la economía argentina revelan un importante crecimiento económico hasta el año 2011, con excepción del año 2009 afectado por la crisis económica mundial iniciada en el año 2008 (IAMC, 2009). Posterior al año 2011, la economía muestra un período de bajo o negativo crecimiento y una fuerte aceleración del proceso inflacionario.

\section{Metodología}

\section{III.I. Datos}

La población objeto de este estudio son todas las empresas manufactureras autorizadas a cotizar en el Mercado de Valores de Buenos Aires en el período 2005 a 2016, excluidas las de capital extranjero. El estudio incluye, solamente, empresas de la industria manufacturera para que la muestra sea homogénea, debido a que el capital corriente difiere de acuerdo con el sector de la industria (Chiou y Cheng, 2006; Filbeck y Krueger, 2005). No obstante, el sector manufacturero está integrado por un conjunto de industrias con sus propias características.

Las observaciones de la muestra están constituidas por datos de los estados financieros anuales consolidados de dichas empresas, elaborados bajo Normas Contables Profesionales de la Federación Argentina de Consejos Profesionales de Ciencias Económicas (FACPCE) y las Normas Internacionales de Información Financiera (NIIF). La muestra inicial es de 450 observaciones y después de eliminar los datos atípicos (outliers) quedaron 406 observaciones que corresponden a 47 empresas. Han sido considerados outliers aquellas observaciones que se alejan 3 veces de la desviación estándar de la media. Una de las limitaciones del análisis es que las cifras de los estados financieros corresponden a una economía inflacionaria y no se encuentran ajustados por inflación. Sin embargo, una de las características del SEM es que al emplear múltiples medidas por cada variable permite disminuir los errores de medición.

\section{III.II. Modelos desarrollados}

La relación entre capital corriente con la rentabilidad y la solvencia es analizada por la metodología SEM. Esta permite examinar simultáneamente múltiples y conexas relaciones de dependencia, y es particularmente útil cuando una variable dependiente se convierte en variable independiente en posteriores relaciones de dependencia. Además, muchas de las variables afectan a cada una de las variables dependientes, pero con efectos distintos. Otra característica, es que sirve para evaluar la relación entre constructos no observables, denominadas variables latentes. La variable latente es un constructo supuesto que puede ser medido mediante un conjunto de variables observables. Esta técnica posibilita emplear múltiples medidas para una 
variable latente y de esa forma controla el error específico de cada variable. Los resultados del modelo muestran la correlación entre las variables, pero esto no prueba la relación de causalidad, la cual debe ser deducida de la teoría. Esta metodología proviene del ámbito de la psicología y ha sido aplicada a la investigación contable (Jairo, 2008; Bou y Satorra, 2007; Titman y Wessels, 1988). Asimismo, Baser, Gokten, Kucukkocaoglu y Ture (2016) la aplicaron al estudio de liquidez y rentabilidad. Para la aplicación de la metodología fue utilizado el diagrama para la construcción y medición de SEM del software Stata.

El modelo propuesto para este trabajo está compuesto por variables que en conjunto miden y representan la liquidez, rentabilidad y solvencia, tal como lo muestra la figura 2 .

Figura 2: Modelo estructural

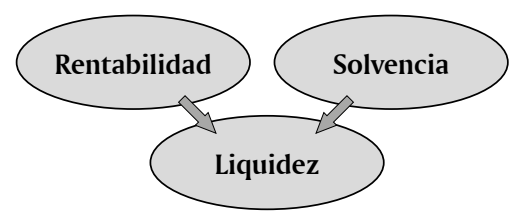

Fuente: Elaboración propia.

Las variables utilizadas en el estudio son:

a) Rentabilidad:

Rentabilidad del patrimonio neto:

$$
R P N=\frac{G N}{P N(p)}
$$

Rentabilidad del activo:

$$
R A=\frac{G a i I}{A C T(p)}
$$

Margen neto de ventas:

$$
M g=\frac{G N}{V T A}
$$

Rotación de activos:

$$
R O T A=\frac{V T A}{A C T(p)}
$$

Incremento de ventas:

$$
\Delta V T A_{t}=\frac{V T A_{t}}{V T A_{t-1}}-1
$$


Donde:

GN: ganancia neta.

PN: patrimonio neto.

Gail: ganancia antes de intereses e impuestos.

ACT: activos.

VTA: ventas netas.

ROTA: rotación de activos.

b) Liquidez:

Las variables intervinientes en este estudio son:

Liquidez corriente:

$$
L C=\frac{A C}{P C}
$$

La liquidez efectiva neta no resulta ser significativa para el modelo, pero sí cuando se incluyen a ésta las deudas comerciales, la cual es conocida como razón de efectivo.

Razón de efectivo:

$$
L Q=\frac{E F+E E}{D C+D F a C P}
$$

Plazo de cobranza:

$$
P C=\frac{C R(p) X 365}{V T A}
$$

Siendo el promedio:

$$
(p)=\frac{S I+S F}{2}
$$

Plazo de almacenamiento:

$$
P A=\frac{B C(p) X 365}{C V}
$$

Plazo de pago deudas comerciales:

$$
P P=\frac{D C(p) X 365}{C}
$$

Ciclo de conversión de efectivo:

$$
C C E=P C+P A-P P
$$

Liquidez requerida:

$$
L R=\frac{C R(p)+B C(p)}{D C(p)}
$$


Terreno, D., Pérez, J. y Sattler, S. La relación entre liquidez, rentabilidad y solvencia...

Donde:

EF: efectivo.

EE: equivalente de efectivo (inversiones de corto plazo).

CR: créditos por ventas.

BC: bienes de cambio.

DC: deudas comerciales.

ACT: activos.

AC: activo corriente.

PC: pasivo corriente.

$\mathrm{CR}(\mathrm{p})$ : créditos por ventas promedio.

$\mathrm{BC}(\mathrm{p})$ : bienes de cambio promedio.

$\mathrm{CV}$ : costo de ventas.

DC (p): deudas comerciales promedio.

C: compras.

c) Solvencia:

$$
E L P=\frac{P N C}{P N}
$$

PNC: pasivo no corriente.

\section{III.III. Modelo 1: por niveles}

El modelo por niveles se refiere a la medición de los ratios por las cifras que surgen en el Estado de Situación Patrimonial acumuladas al cierre, y en el Estado de Resultados acumulados por cada año. Para la selección de las variables del modelo se efectúa un estudio exploratorio por el análisis factorial. La tabla 3 informa los datos obtenidos en el primer factor y la tabla $\mathrm{N}^{\circ} 4$ muestra los coeficientes de correlación de Spearman.

Tabla 3: Análisis de componentes principales

\begin{tabular}{cc}
\hline $\mathbf{n}=406$ & Comp1 \\
\hline Valor propio & 3,3184 \\
Proporción & 0,4253 \\
\hline RPN & 0,5790 \\
RA & 0,5861 \\
Mg & 0,6201 \\
ROTA & 0,0911 \\
DVTA & 0,0966 \\
LC & 0,6809 \\
LQ & 0,2106 \\
LR & 0,5752
\end{tabular}




\begin{tabular}{cc}
\hline $\mathbf{n}=406$ & Comp1 \\
\hline CCE & 0,7943 \\
PC & 0,0861 \\
PA & 0,6313 \\
PP & $(0,4531)$ \\
ELP & $(0,3954)$ \\
\hline
\end{tabular}

Fuente: Elaboración propia.

Tabla 4: Coeficientes de correlación de Spearman: modelo 1

\begin{tabular}{lllllllllllll}
\hline & RPN & RA & Mg & ROTA & LC & LQ & LR & CCE & PC & PA & PP & ELP \\
\hline RPN & 1,00 & & & & & & & & & & & \\
RA & 0,84 & 1,00 & & & & & & & & & \\
Mg & 0,83 & 0,73 & 1,00 & & & & & & & & \\
ROTA & 0,31 & 0,31 & $-0,05$ & 1,00 & & & & & & & \\
LC & 0,33 & 0,39 & 0,46 & 0,10 & 1,00 & & & & & & & \\
LQ & 0,23 & 0,29 & 0,32 & $-0,03$ & 0,58 & 1,00 & & & & & \\
LR & 0,18 & 0,24 & 0,31 & 0,03 & 0,74 & 0,40 & 1,00 & & & & & \\
CCE & 0,11 & 0,16 & 0,23 & $-0,03$ & 0,68 & 0,30 & 0,91 & 1,00 & & & & \\
PC & $-0,05$ & $-0,18$ & $-0,07$ & $-0,08$ & 0,10 & $-0,07$ & 0,11 & 0,24 & 1,00 & & & \\
PA & 0,13 & 0,20 & 0,24 & $-0,08$ & 0,52 & 0,18 & 0,66 & 0,78 & $-0,04$ & 1,00 & & \\
PP & $-0,05$ & $-0,04$ & $-0,11$ & $-0,20$ & $-0,44$ & $-0,35$ & $-0,61$ & $-0,40$ & 0,34 & $-0,02$ & 1,00 & \\
ELP & $-0,26$ & $-0,25$ & $-0,30$ & $-0,33$ & $-0,37$ & $-0,15$ & $-0,28$ & $-0,25$ & $-0,03$ & $-0,24$ & 0,18 & 1,00 \\
\hline
\end{tabular}

Fuente: Elaboración propia.

De acuerdo con el análisis factorial son excluidas la rotación de los activos $(0,0911)$, el crecimiento de las ventas $(0,0966)$ y el plazo de cobranza $(0,0861)$ por el bajo peso factorial. Por otra parte, la correlación entre el ciclo de conversión en efectivo y la liquidez requerida es superior 0,85 (Kline, 2011), por lo cual ambas variables brindan la misma información al modelo. De ambas variables se excluye el ciclo de conversión de efectivo debido a que la liquidez requerida mostró un mejor ajuste del modelo. Por lo anterior, el modelo se define de la siguiente forma:

$$
\begin{gathered}
\text { Rentabilidad }=f(R P N, R A, M g) \\
\text { Capital corriente }=f(L C, L Q, L R, P A, P P) \\
\text { Solvencia }=E L P
\end{gathered}
$$

La solvencia es representada únicamente por el ratio de endeudamiento a largo plazo debido a que los otros ratio de la solvencia no se ajustaron al modelo. 
Terreno, D., Pérez, J. y Sattler, S. La relación entre liquidez, rentabilidad y solvencia...

\section{III.IV. Modelo 2: por diferencias}

Este modelo define las variables de la liquidez y el endeudamiento por los cambios de un año con respecto al anterior, tal como el estudio de Chiou y Cheng (2006); por ejemplo, en caso de la LC:

$$
\Delta L C_{t}=\frac{L C_{t}}{L C_{t-1}}-1
$$

La tabla 5 muestra los coeficientes de correlación de Spearman del modelo por diferencias; ninguno de los coeficientes muestra una correlación superior 0,85 . El $\triangle$ PA fue excluido porque mostró no ajustarse al modelo.

Tabla 5: Coeficientes de correlación de Spearman: modelo 2

\begin{tabular}{lccccccccc}
\hline & RPN & RA & Mg & $\Delta$ LC & $\Delta$ LQ & $\Delta$ LR & $\Delta$ PC & $\Delta$ PP & $\Delta$ ELP \\
\hline RPN & 1,00 & & & & & & & & \\
RA & 0,84 & 1,00 & & & & & & & \\
Mg & 0,83 & 0,73 & 1,00 & & & & & & \\
$\Delta$ LC & 0,16 & 0,16 & 0,12 & 1,00 & & & & & \\
$\Delta$ LQ & 0,06 & 0,12 & 0,04 & 0,40 & 1,00 & & & & \\
$\Delta$ LR & 0,12 & 0,16 & 0,02 & 0,29 & 0,11 & 1,00 & & & \\
$\Delta$ PC & $-0,13$ & $-0,08$ & $-0,09$ & $-0,06$ & $-0,15$ & $-0,50$ & 1,00 & & \\
$\Delta$ PP & $-0,08$ & $-0,08$ & $-0,02$ & $-0,17$ & $-0,15$ & $-0,50$ & 0,33 & 1,00 & \\
$\Delta$ ELP & $-0,07$ & $-0,06$ & $-0,03$ & 0,33 & 0,06 & 0,07 & $-0,12$ & $-0,08$ & 1,00 \\
\hline
\end{tabular}

Fuente: Elaboración propia.

La tabla 6 muestra los estadísticos descriptos de las variables utilizadas en los modelos 1 y 2 . Las variables definidas como incrementos y LQ son las que muestran una mayor dispersión de los datos.

Tabla 6: Estadísticos descriptivos

\begin{tabular}{lccc}
\hline Variable & Media & Mediana & Desv. \\
\hline RPN & 0,071 & 0,100 & 0,236 \\
RA & 0,128 & 0,125 & 0,106 \\
Mg & 0,079 & 0,040 & 0,090 \\
LC & 1,602 & 1,438 & 0,787 \\
LQ & 0,680 & 0,274 & 1,471 \\
LR & 3,055 & 2,634 & 2,512 \\
PA & 91,549 & 88,000 & 54,586 \\
PP & 71,520 & 64,000 & 37,050 \\
$\Delta$ LC & $-0,048$ & $-0,045$ & 0,379 \\
$\Delta$ LQ & $-0,553$ & $-0,013$ & 1,017
\end{tabular}




\begin{tabular}{lccc}
\hline Variable & Media & Mediana & Desv. \\
\hline$\Delta \mathrm{LR}$ & $-0,269$ & $-0,090$ & 1,622 \\
$\Delta \mathrm{PP}$ & 5,0172 & 2,000 & 28,251 \\
$\Delta \mathrm{ELP}$ & 0,006 & $-0,002$ & 0,075 \\
\hline
\end{tabular}

Fuente: Elaboración propia.

\section{Resultados}

\section{IV.I. Modelo 1: por niveles}

La literatura recomienda emplear múltiples indicadores para evaluar la bondad del ajuste del modelo (Hu y Bentler, 1995). La bondad del ajuste se refiere a la exactitud de los supuestos del modelo especificado para determinar si el modelo es correcto y sirve como aproximación al fenómeno real, precisando así su poder de predicción. La tabla $\mathrm{N}^{\circ} 7$ muestra el ajuste del modelo estructural. Todos los índices se ubican en la zona de ajuste "aceptable" a excepción $\chi^{2} / \mathrm{df}$, pero está muy cercano a 3 .

Tabla 7: Índices de ajuste del modelo estructural: modelo 1

\begin{tabular}{lccc}
$\mathrm{n}=406$ & & \\
\hline \multicolumn{1}{c}{ Medidas de ajuste } & Medida obtenida & Buen ajuste (*) & Aceptableajuste (*) \\
\hline Chi-cuadrado $\left(\chi^{2}\right)$ & 78,41 & $0 \leq \chi^{2} \leq 2 \mathrm{df}$ & $2 \mathrm{df}<\chi^{2} \leq 3 \mathrm{df}$ \\
Grados de libertad (df) & 23 & & \\
p-valor & 0,00 & $0,05<\mathrm{p} \leq 1,00$ & $0.01 \leq \mathrm{p}<0,05$ \\
$\chi^{2} / \mathrm{df}$ & 3,41 & $0 \leq \chi^{2} / \mathrm{df} \leq 2$ & $2<\chi^{2} / \mathrm{df} \leq 3$ \\
RMSEA & 0,077 & $0 \leq \mathrm{RMSEA} \leq 0,05$ & $0,05 \leq \mathrm{RMSEA} \leq 0,08$ \\
SRMR & 0,078 & $0 \leq \mathrm{SRMR} \leq 0,05$ & $0,05 \leq$ SRMR $\leq 0,10$ \\
CFI & 0,952 & $0,97<\mathrm{p} \leq 1,00$ & $0,95 \leq \mathrm{p}<0,97$ \\
\hline
\end{tabular}

(*) Schermelleh-Engel, Moosbrugger y Müller (2003).

Fuente: Elaboración propia.

Tabla 8: Coeficientes estandarizados: modelo 1

\begin{tabular}{lcccccc}
$\mathrm{n}=406$ & & & & & & \\
\hline Liquidez y rentabilidad & & $\mathbf{0 , 3 4}$ & $\mathbf{0 , 0 0}$ & & & \\
\hline Endeudamiento y liquidez & & $(0,25)$ & 0,00 & & & \\
\hline \multirow{4}{*}{ Rentabilidad } & $\mathrm{RPN}$ & 0,89 & 0,00 & 0,30 & 0,00 & 0,21 \\
& $\mathrm{RA}$ & 0,83 & 0,00 & 1,21 & 0,00 & 0,32 \\
& $\mathrm{Mg}$ & 0,82 & 0,00 & 0,42 & 0,00 & 0,34
\end{tabular}


Terreno, D., Pérez, J. y Sattler, S. La relación entre liquidez, rentabilidad y solvencia...

\begin{tabular}{|c|c|c|c|c|c|c|}
\hline & Variable & Coef. & $P>z$ & $\alpha$ & $P>Z$ & Error \\
\hline \multirow{5}{*}{ Liquidez } & LC & 0,96 & 0,00 & 2,35 & 0,00 & 0,01 \\
\hline & LQ & 0,27 & 0,00 & 0,53 & 0,00 & 0,93 \\
\hline & LR & 0,48 & 0,00 & 1,35 & 0,00 & 0,77 \\
\hline & PA & 0,47 & 0,00 & 1,81 & 0,00 & 0,78 \\
\hline & PP & $(0,34)$ & 0,00 & 1,42 & 0,00 & 0,89 \\
\hline PP y PA & & 0,20 & 0,00 & & & \\
\hline PA y LR & & 0,22 & 0,00 & & & \\
\hline PP Y LR & & $(0,30)$ & 0,00 & & & \\
\hline
\end{tabular}

Fuente: Elaboración propia.

La Tabla 8 y la Figura 3 informan los resultados obtenidos del modelo 1. Los coeficientes de todas las variables observables de la rentabilidad son todos significativos y positivos. El mayor valor corresponde a la RPN $(0,89)$, seguido de la $\mathrm{RA}(0,83)$ y $\mathrm{Mg}(0,82)$ con valores muy próximos; por análisis factorial se ha descartado la ROTA y $\triangle$ VTA.

Figura 3: Modelo estructural: por niveles

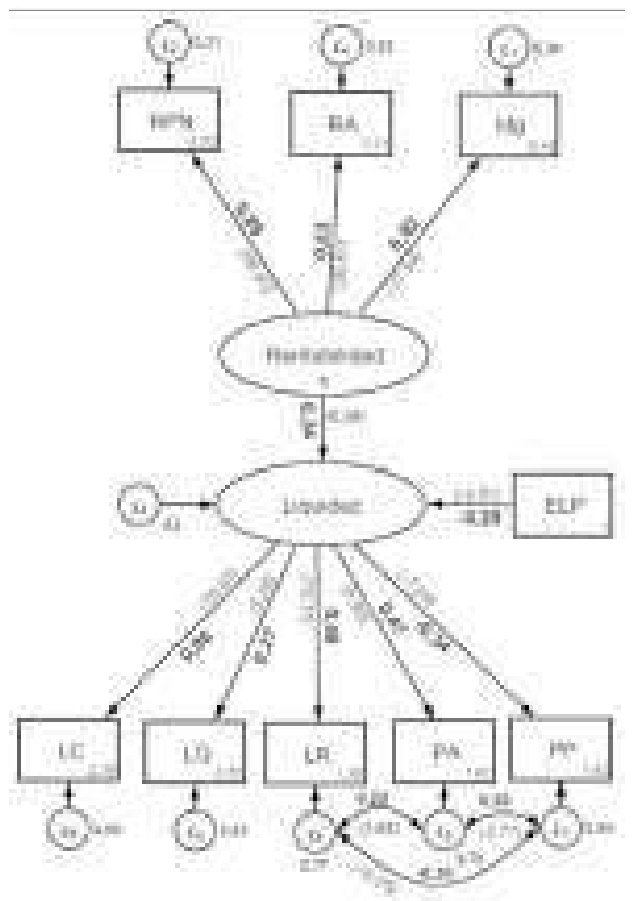

Fuente: Elaboración propia (Output Stata). 
Los coeficientes de las variables de la liquidez son significativos y positivos, a excepción del PP negativo. El mayor coeficiente corresponde a la LC $(0,96)$ que representa la totalidad del capital corriente, el cual es determinado por la LR $(0,48)$ que mide el capital corriente requerido y la LQ $(0,27)$ que mide los componentes financieros del capital corriente con las deudas comerciales. El menor valor del coeficiente de la LQ se explica por una mayor volatilidad de sus componentes. La LR es determinado por el PA $(0,47)$ y PP $(-0,34)$, componentes del ciclo de conversión de efectivo. El plazo de almacenamiento depende de la duración del proceso productivo y de la política de inventarios, mientras que, el plazo de pago de deudas comerciales muestra la importancia de la financiación de los proveedores. La covarianza del PA $(0,22)$ y PP $(-0.30)$ con LR son significativas, la primera positiva y la segunda negativa, confirmando la relación con la liquidez requerida. La covarianza entre el PA y PP $(0.20)$ es positiva y significativa, lo que implica que existe algún grado de relación entre la política de inventarios y la financiación que conceden los proveedores. El plazo de cobranza de acuerdo con el análisis factorial no surge como un determinante de la liquidez requerida. El nivel de créditos no acompaña la evolución del resto de los componentes de los activos y pasivos corrientes, éstos dependen, principalmente, de la política de ventas, la gestión de cobranza y las expectativas inflacionarias.

La relación entre la liquidez y rentabilidad $(0,34)$ es significativa y positiva, las empresas con mayor capital corriente tienen una mayor rentabilidad. Los recursos generados por la empresa son aplicados a la cancelación de pasivo, a inversiones corrientes y a aumentar los inventarios. Esto obedece a la visión cortoplacista del empresario argentino que, como consecuencia del contexto turbulento, es renuente a realizar inversiones a largo plazo y las canaliza en el corto plazo (De Pablo, 2010). Las empresas prefieren una mayor flexibilidad financiera para enfrentar futuras crisis y, además, los inventarios son una manera de protegerse contra la inflación.

La relación entre la liquidez y endeudamiento a largo plazo $(-0.25)$ es significativa y negativa. Las empresas con un mayor endeudamiento de largo plazo tienen un menor capital corriente, lo cual podría obedecer a dos cuestiones. Por una parte, ante la necesidad de financiamiento externo la empresa recurre tanto a deudas comerciales como a deudas financieras de corto y largo plazo. Por otra parte, el costo de pasivo y la necesidad de fondos para la devolución hace que la empresa reduzca al mínimo los activos corrientes y en particular los inventarios.

\section{IV.II. Modelo 2: por diferencias}

La Tabla 9 muestra el ajuste del modelo estructural, el valor de SRMR se ubica en la zona de "buen" ajuste y, la RMSEA, el CFI y la $\chi^{2} / \mathrm{df}$ se ubican en la 
Terreno, D., Pérez, J. y Sattler, S. La relación entre liquidez, rentabilidad y solvencia...

zona de ajuste "aceptable" pero casi en el límite con la zona de "buen ajuste", por lo que se puede considerar un muy buen ajuste del modelo.

Tabla 9: Índices de ajuste del modelo estructural: modelo 2

\begin{tabular}{lccc}
$\mathrm{n}=406$ & & & \\
\multicolumn{1}{c}{ Medidas de ajuste } & Medida & Buen & Aceptable \\
\hline & obtenida & ajuste (*) & ajuste (*) $^{*}$ \\
Chi-cuadrado $\left(\chi^{2}\right)$ & 51,20 & $0 \leq \chi^{2} \leq 2 \mathrm{df}$ & $2 \mathrm{df}<\chi^{2} \leq 3 \mathrm{df}$ \\
Grados de libertad (df) & 25 & & \\
p-valor & 0,002 & $0,05<\mathrm{p} \leq 1,00$ & $0.01 \leq \mathrm{p}<0,05$ \\
$\chi^{2} / \mathrm{df}$ & 2,048 & $0 \leq \chi^{2} / \mathrm{df} \leq 2$ & $2<\chi^{2} / \mathrm{df} \leq 3$ \\
RMSEA & 0,051 & $0 \leq \mathrm{RMSEA} \leq 0,05$ & $0,05 \leq \mathrm{RMSEA} \leq 0,08$ \\
SRMR & 0,048 & $0 \leq$ SRMR $\leq 0,05$ & $0,05 \leq \mathrm{SRMR} \leq 0,10$ \\
CFI & 0,968 & $0,97<\mathrm{p} \leq 1,00$ & $0,95 \leq \mathrm{p}<0,97$ \\
\hline
\end{tabular}

(*) Schermelleh-Engel, Moosbrugger y Müller (2003).

Fuente: Elaboración propia.

Tabla 10: Coeficientes estandarizados: modelo 2

\begin{tabular}{lcccccc}
$\mathrm{n}=406$ & Variable & Coef. & $P>z$ & $\alpha$ & $P>z$ & Error \\
\hline Rentabilidad y liquidez & & 0,16 & 0,00 & & & \\
Endeudamiento y liquidez & & 0,40 & 0,00 & & & \\
& $\mathrm{RPN}$ & 0,90 & 0,00 & 0,30 & & 0,18 \\
Rentabilidad & $\mathrm{RA}$ & 0,82 & 0,00 & 1,21 & & 0,32 \\
& $\mathrm{Mg}$ & 0,80 & 0,00 & 0,42 & & 0,36 \\
& $\Delta \mathrm{LC}$ & 1,00 & 0,00 & $-0,13$ & 0,01 & 0,01 \\
Liquidez & $\Delta \mathrm{LQ}$ & 0,22 & 0,00 & $-0,06$ & 0,24 & 0,95 \\
& $\Delta \mathrm{LR}$ & 0,20 & 0,01 & $-0,17$ & 0,00 & 0,96 \\
& $\Delta \mathrm{PC}$ & $-0,15$ & 0,00 & 0,21 & 0,00 & 0,98 \\
& $\Delta \mathrm{PP}$ & $-0,18$ & 0,00 & 0,18 & 0,00 & 0,97 \\
$\Delta \mathrm{PC}$ y $\triangle \mathrm{PP}$ & & 0,23 & 0,00 & & & \\
\hline
\end{tabular}

Fuente: Elaboración propia.

La Tabla 10 y la Figura 4 informan los resultados obtenidos del modelo. Los coeficientes de las variables de liquidez son todos significativos. El mayor valor corresponde al $\Delta \mathrm{LC}(1,00)$, los coeficientes de $\Delta \mathrm{LQ}(0,22)$ y $\Delta \mathrm{LR}(0,20)$ son positivos, si bien bastante bajos en relación con $\triangle \mathrm{LC}$. Los coeficientes de $\triangle \mathrm{PC}(-0,15)$ y $\triangle \mathrm{PP}(-0,18)$ son negativos y por lógica bajos; adicionalmente, 
muestran una covarianza positiva y significativa $(0,23)$. Los coeficientes tienen la dirección esperada, a excepción del cambio en el plazo de cobranza que tiene una relación negativa; esto puede ser como consecuencia de que el aumento del plazo de cobranza es financiado por un mayor plazo de pago de deudas comerciales.

Las variables de la rentabilidad son significativas y positivas, y con valores similares al modelo 1; el análisis por los cambios en el nivel de rentabilidad, no tabulados, no resultaron ser significativos en relación con los cambios de la liquidez. La relación entre la liquidez y rentabilidad $(0,16)$ es positiva y significativa, pero con un coeficiente menor al del modelo 1 que relaciona el nivel rentabilidad y liquidez. La relación entre el cambio en la liquidez y $\Delta$ ELP $(0,40)$ es significativa y exhibe un valor importante; pero contrario al modelo 1 muestra un signo positivo. El cambio en el endeudamiento de largo plazo afecta los cambios del capital de trabajo de forma positiva, ya sea porque los fondos obtenidos de largo plazo son invertidos en activos corrientes o por la renovación de deudas de corto plazo a largo plazo.

Figura 4: Modelo estructural: modelo 2

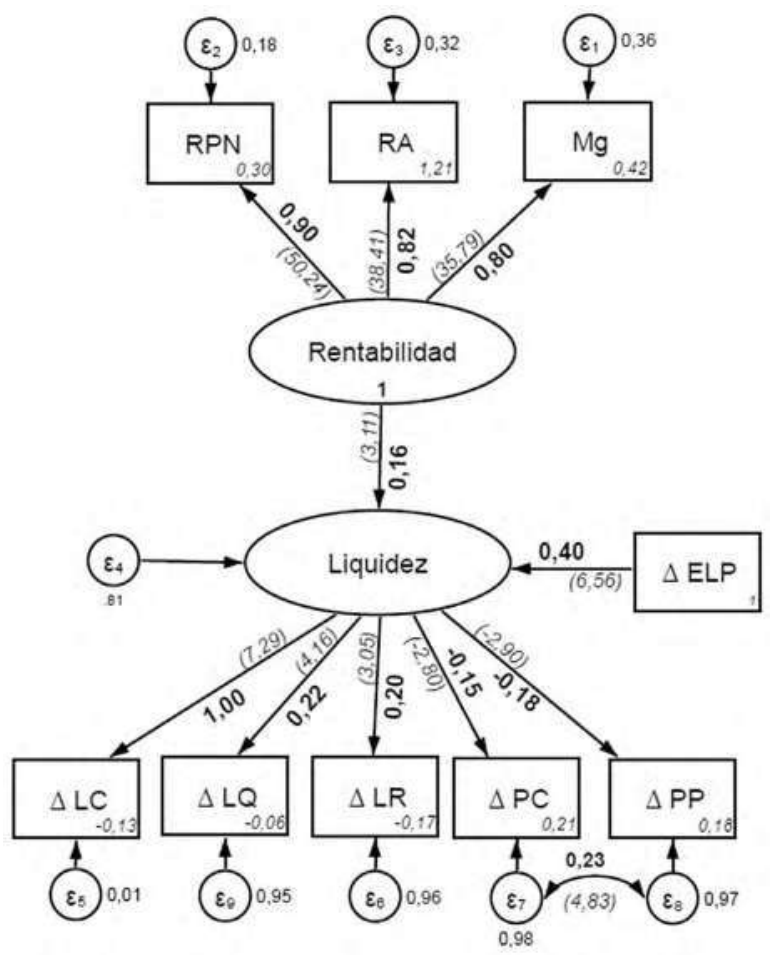

Fuente: Elaboración propia (Output Stata). 
Terreno, D., Pérez, J. y Sattler, S. La relación entre liquidez, rentabilidad y solvencia...

\section{Conclusiones}

En el estudio se efectúa un análisis de la liquidez con la rentabilidad y endeudamiento por niveles, y por las diferencias entre el año actual y el anterior de los ratios de liquidez y endeudamiento. La muestra de estudio son las empresas manufactureras listadas en el Mercado de Valores de Buenos Aires en el período 2005 a 2016. La metodología aplicada de ecuaciones estructurales permite un enfoque integrado del problema y reduce los errores de medición.

En el análisis por niveles, la relación entre la liquidez y rentabilidad muestra que las empresas con mayor capital corriente tienen una mayor rentabilidad. Los recursos generados por un mayor nivel de rentabilidad son aplicados al capital corriente, ya sea a la cancelación del pasivo, a inversiones corrientes y a aumentar los inventarios. Esto obedece a una actitud cortoplacista del empresario argentino debido a la incertidumbre de la economía y la necesidad de contar una mayor flexibilidad financiera para afrontar las crisis recurrentes. En este contexto, la inversión en inventarios es parte de la estrategia para protegerse de la inflación. El análisis muestra que la liquidez corriente es afectada por la liquidez requerida y la razón de efectivo, pero esta última en menor medida. La liquidez requerida es determinada por el plazo de almacenamiento y el plazo de pago de deudas comerciales, mientras que el plazo de cobranza no mostró ser relevante. En el análisis del modelo por diferencias, la relación de las variaciones de la liquidez con el nivel de rentabilidad es positiva y significativa, pero si bien mantiene el mismo signo, exhibe una menor importancia que en análisis por niveles.

La relación entre liquidez y solvencia, esta última medida por el ratio de endeudamiento a largo plazo, tienen una relación negativa. Las empresas con mayor endeudamiento de largo plazo tienen un menor capital corriente, lo cual podría obedecer a dos cuestiones. Por una parte, ante la necesidad de financiamiento externo se recurre a diversas fuentes de financiamiento, lo cual incluye deudas de corto plazo que reducen la liquidez. Por otro lado, el mayor endeudamiento, con el costo y riesgo que implica, obliga a reducir al mínimo los activos corrientes y en particular los inventarios. Pero, la relación entre los cambios del capital corriente y los cambios del endeudamiento de largo plazo es positiva, ya sea porque los fondos prestados de largo plazo son invertidos en activos corrientes o por la refinanciación de deudas del corto al largo plazo.

En general, los resultados del estudio contradicen a una gran parte de la bibliografía que sostiene una relación negativa entre liquidez y rentabilidad, y es coincidente con la mayor parte que sostiene una relación negativa entre liquidez y endeudamiento. Además, el estudio amplía las conclusiones de estudios anteriores, ya que demuestra que la relación entre los cambios del capital corriente y endeudamiento de largo plazo es positiva. Por 
último, confirma que la simple apreciación, a priori, de la relación entre los componentes de los estados financieros sin recurrir a la evidencia empírica puede ser equivocada. Como un aporte para futuras investigaciones, este estudio debería extenderse a otro tipo de actividades, tales como servicios, constructoras, etc.

\section{Referencias bibliográficas}

Afza, T. y Nazir, M. S. (2007). Is it better to be aggressive or conservative in managing working capital. Journal of quality and technology management, 3(2), 11-21.

Anuarios del Instituto Argentino del Mercado de Capitales-IAMC (2009). Recuperado de: http: //www.iamc.sba.com.ar/informes/informe_anuario/.

Azeem, M. M. y Marsap, A. (2015). Determinant factors and working capital requirement. International Journal of Economics and Finance, 7(2), 280-292. Doi:/10.5539/ijef.v7n2p280

Baser, F., Gokten, S., Kucukkocaoglu, G. y Ture, H. (2016). Liquidity-profitability Tradeoff Existence in Turkey: an Empirical Investigation under Structural Equation Modeling. Copernican Journal of Finance \& Accounting, 5(2), 27-44. Doi:/10.12775/CJFA.2016.013

Bou, J. C. y Satorra, A. (2007). The persistence of abnormal returns at industry and firm levels: Evidence from Spain. Strategic Management Journal, 28(7), 707-722. Doi:/10.1002/smj.586

Brealey, R., Myers, S., y Allen, F. (2006). Principios de Finanzas Corporativas. Madrid: McGrawHill.

Chiou, J. R. y Cheng, L. (2006). The Determinants of Working Capital Management. Journal of American Academy of Business, 10(1), 149-155.

Deloof, M. (2003). Does working capital management affect profitability of Belgian firms? Journal of Business Finance \& Accounting, 30(3\&4), 573-587. Doi: 10.1111/14685957.00008

De Pablo, J. C. (2010). 200 años de economía argentina: (nunca un momento aburrido). Argentina: Ediciones B.

Eljelly, A. M. (2004). Liquidity-profitability tradeoff: An empirical investigation in an emerging market. International journal of commerce and management, 14(2), 48-61. Doi: $10.1108 / 10569210480000179$

Filbeck, G. y Krueger, T. M. (2005). An analysis of working capital management results across industries.American Journal of Business, 20(2), 11-20. Doi:/10.1108/19355181200500007

García-Teruel, P. y Martínez-Solano, P. (2007). Effects of working capital management on SME profitability. International Journal of managerial finance, 3(2), 164-177. Doi: $10.1108 / 17439130710738718$

Gill, A. (2011). Factors that influence working capital requirements in Canada. Economics and Finance Review, 1(3), 30-40.

Gill, A., Biger, N. y Mathur, N. (2010). The relationship between working capital management and profitability: Evidence from the United States. Business and economics journal, 10(1), 1-9.

Gitman, L. J. y Sachdeva, K. S. (1982). A framework for estimating and analyzing the required working capital investment. Review of Financial Economics, 17(3), 36. 
Terreno, D., Pérez, J. y Sattler, S. La relación entre liquidez, rentabilidad y solvencia...

Hu, L. y Bentler, P. M. (1999). Cutoff criteria for fit indexes in covariance structure analysis: Conventional criteria versus new alternatives. Structural equation modeling: $a$ multidisciplinary journal, 6, 1-55.

Jairo, I. (2008). The use of structural equation modelling (SEM) in capital structure empirical analysis. KCA Journal of Business Management, 1(1). Doi: 10.4314/kjbm.v1i1.43819

Kadayifci, S. y Coskun, A. (2017). An Analysis of the Factors Determining the Working Capital Requirement for Non-Financial Companies. International Conference on Economic and Social Studies. Doi: 10.14706/icesos 1724

Kline, R.B. (2011). Principles and practice of structural equation modeling (3rd Ed.). New York, NY: The Guilford Press.

Lazaridis, I. y Tryfonidis, D. (2006). Relationship between working capital management and profitability of listed companies in the Athens stock exchange. Journal of financial management and analysis, 19(1).

Mandujano Herrera, R. y Navarro Orihuela, J. (2015). Factores determinantes del capital de trabajo en empresas manufactureras peruanas y chilenas listadas en el mercado integrado latinoamericano. Working paper de la Universidad del Pacífico. Recuperado de: http://repositorio.up.edu.pe/handle/11354/1729

Maradi, M., Salehi, M. y Arianpoor, A. (2012). A comparison of working capital management of chemical and medicine listed companies in Tehran Stock Exchange. International Journal of Business and Behavioral Science, 2(5), 62-78.

Naser, K., Nuseibeh, R. y Al-Hadeya, A. (2013). Factors influencing corporate working capital management: Evidence from an emerging economy. Journal of Contemporary Issues in Business Research, 2(1), 11-30.

Palombini, N. V. N. y Nakamura, W. T. (2012). Key factors in working capital management in the Brazilian market. Revista de Administração de Empresas, 52(1), 55-69. Doi:/10.1590/ S0034-75902012000100005

Raheman, A. y Nasr, M. (2007). Working capital management and profitability-case of Pakistani firms. International review of business research papers, 3(1), 279-300. DOI: 10.12691/jbms-6-1-4

Raykov, E. (2017). The liquidity-profitability trade-off in Bulgaria in terms of the changed financial management functions during crisis. Management: journal of contemporary management issues, 22(1), 135-156. DOI: 10.30924/mjcmi/2017. 22.1.135

Saarani, A. N. y Shahadan, F. (2012). The determinant factors of working capital requirements for Enterprise 50 (E50) firms in Malaysia: Analysis using Structural Equation Modelling. Scottish Journal of Arts, Social Sciences and Scientific Studies, 5(2), 52-66.

Schermelleh-Engel, K., Moosbrugger, H. y Muiller, H. (2003). Evaluating the fit of structural equation models: Tests of significance and descriptive goodness-of-fit measures. Methods of psychological research online, 8(2), 23-74.

Shin, H. H. y Soenen, L. (1998). Efficiency of working capital management and corporate profitability. Financial practice and education, 8, 37-45.

Shubita, M. F. y Alsawalhah, J. M. (2012). The relationship between capital structure and profitability. International Journal of Business and Social Science, 3(16). 
Shulman, J. M. y Cox, R. (1985). An integrative approach to working capital management. Journal of cash management, 5(6), 64-68.

Titman, S. y Wessels, R. (1988). The determinants of capital structure choice. The Journal of finance, 43(1), 1-19.

Vazquez, X. E., Rech, I. J., Miranda, G. J. y Tavares, M. (2017). Convergencias entre la rentabilidad y la liquidez en el sector del agronegocio. Cuadernos de contabilidad, 18(45), 152-165. Doi:/10.11144/Javeriana.cc18-45.crls

Wasiuzzaman, S. y Arumugam, V. C. (2013). Determinants of working capital investment: a study of Malaysian PublicListed firms. Australasian Accounting, Business and Finance Journal, 7(2), 63-83. Doi: 10.14453/aabfj.v7i2.5

Zariyawati, M. A., Annuar, M. N., Taufiq, H. y Rahim, A. A. (2009). Working capital management and corporate performance: Case of Malaysia. Journal of Modern Accounting and Auditing, 5(11), 47. 